\title{
Real-time Movement Image Capturing System Based on Apriori Association and Multiple Calibration Quantization
}

\author{
Shi Feng ${ }^{1}$, HongXia $\mathrm{Hu}^{1}$ \\ ${ }^{1}$ Department of physical Education, Nanchang Institute of Technology, Nanchang, 330099, China \\ shi_feng2014@yeah.net
}

Keywords: Multiple calibrations, Action element, Real-time capture, Triangle image, Relevance, Material library.

\begin{abstract}
In order to the need of meet aerobics technical movement large data processing, it can realize the real-time capture of aerobics video and continuity image, this paper proposes a image processing method based on Apriori correlation and multiple quantitative calibration. The algorithm is verified by the use of aerobics image real-time capture and the analysis of action elements and through the results analysis of image real time processing, we found that images are lower throughput, meeting the requirement of picture fidelity in the process of real-time processing, and good processing timeliness. After quantization processing, aerobics technical continuity action are retained some action element, and the key elements are shown by the form of the triangular cloud, so as to analyze the aerobics technical movements and artistic elements, which provides a new computer method for the construction of the aerobics movement design material library.
\end{abstract}

\section{Introduction}

Due to the development of digital multimedia technology, all kinds of product visual effect gradually begins to bring forth the new through the old by the use of digital multimedia design means, many businesses design very beautiful and good artistic effect visual image [1,2]. In each field modern society, the real time image processing system plays a very important role. Digital image and video information have wide application in various fields, such as military, aerospace, safety monitoring, satellite remote sensing, entertainment, television, medical imaging and so on. With the development of technology and the widespread application, people have increasingly high requirements for the video and image, including clearer image video information resolution, faster and faster processing speed, more variety classification and retrieval etc.[3-5]. This paper uses realtime image processing technology, and combined with Apriori association algorithm to carry on calibration for the aerobics movement image elements. At the same time, through the aerobics image discrete technical elements database obtained by quantizing, the main design process and overall design framework are shown in Figure 1.

Figure 1 shows the process of the aerobics image movement data mining simulation. In order to realize the relevance of data mining, first of all we need to set the support threshold, and then call the Apriori to carry on correlation calculation, the frequent item set can be obtained, finally we can discover association rules [6]. In order to realize the 3D scanning function of image movement, we use multiple scanning head calibration technology to correct the camera angles of the camera, and the use of frequent item sets carry out calibration for the action element database, which can realize the process of image automation processing. 


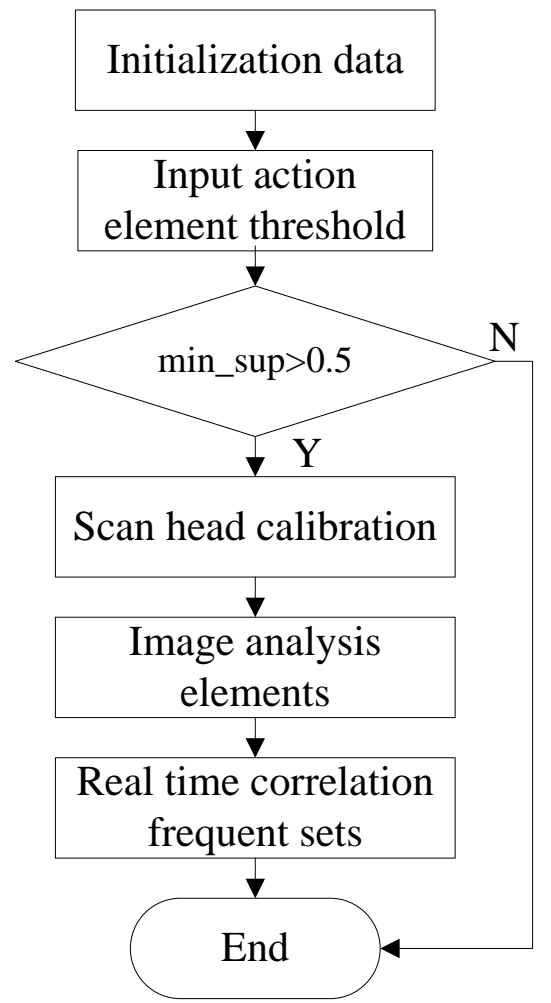

Fig.1: The implementation process of aerobics image

\section{Design of Aerobics Image Real-time Processing Algorithm}

Analysis of the calisthenics image motion is based on image real-time processing algorithm, which involves the processing of large data [7]. Combined with the support degree and confidence degree concept of image real time processing, we can set the association rules of image processing. Assuming that aerobics image is $S$, association rules $A \Rightarrow B$ refers to at the same time appear $A$ and $B$ action frequency, its expression is as follows:

$$
\sup p(A \Rightarrow B)=\frac{\{S: A \cup B \subseteq S, S \in D\} \mid}{|D|} .
$$

Among then, the $D$ represents the aerobics image set in the period of time, the confidence refers to the movement technology elements that have the common ratio both $A$ and $B$.

$$
\operatorname{conf}(A \Rightarrow B)=\frac{|\{S: A \cup B \subseteq S, S \in D\}|}{|S: A \subseteq S, S \in D|} .
$$

Using Apriori algorithm can search the aerobics images, and then we can get the main frequency characteristics of technology elements [8]. Using $k$ itemsets finds the $k+1$ item set, through $L_{i-1} \times L_{i-1}$ connectes to generate candidate sets item $A_{i}$. Assuming $l_{k}(j)$ shows the $L_{i-1}$ items set, if there are

$$
l_{1}[1]=l_{2}[1] \wedge l_{1}[2]=l_{2}[2] \cdots\left(l_{1}[k-1]=l_{2}[k-1]\right) .
$$

Then the connection $l_{1}$ and $l_{2}$ can obtain the result set that is $l_{1}[1], l_{2}[1] \cdots\left(l_{1}[k-1], l_{2}[k-1]\right)$.

$$
l_{1}[k-1]=l_{2}[k-1] \text {. }
$$

Based on the nature of the Fu Liye transform, the $n$ order derivatives of $f(x)$ should satisfy $f^{(n)}(x) \Leftrightarrow(j w)^{n} F(w)$, and then it is extended to fractional order that can be obtained

$$
f^{(a)}(x) \Leftrightarrow D^{a} F(w)=(i w)^{a} F(w) .
$$

According to the search program, we can be the real-time continuous aerobics image to carry on processing, and finally we obtain the analysis results of having the relevance aerobics movement factor, the main algorithm is as follows [9]: 


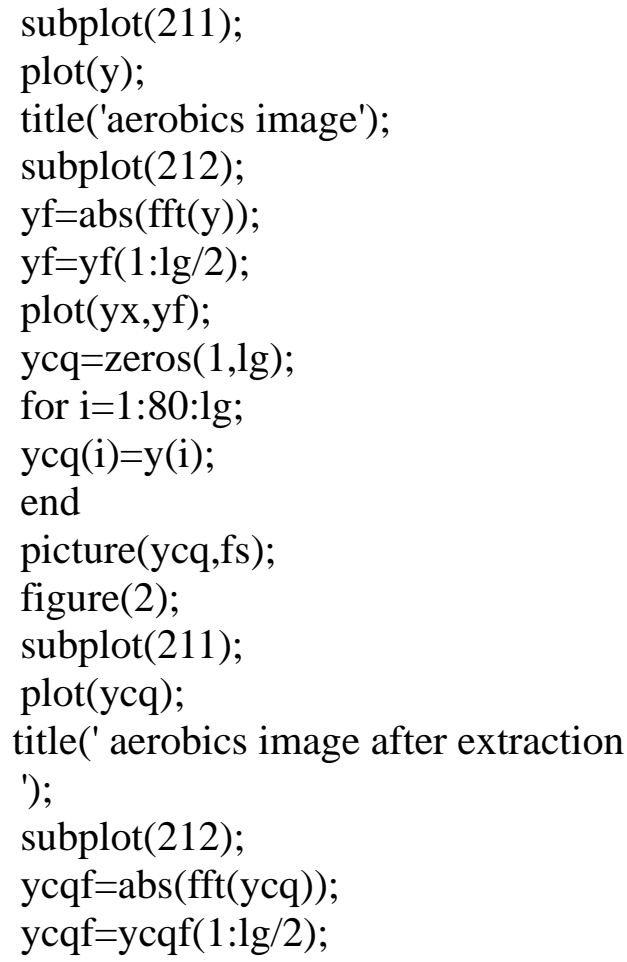

\section{Analysis of Aerobics Technique Action Elements based on Image Processing}

In order to verify the effectiveness and reliability of the real-time image processing algorithm designed in section second, this paper uses 3D image scanning technology, and combined with the Google glasses and multiple calibration technology to carry on the real time image processing of aerobics continuous video, which can get the analysis results of aerobics movement factor $[10,11]$. Google glasses are shown in Figure 2.
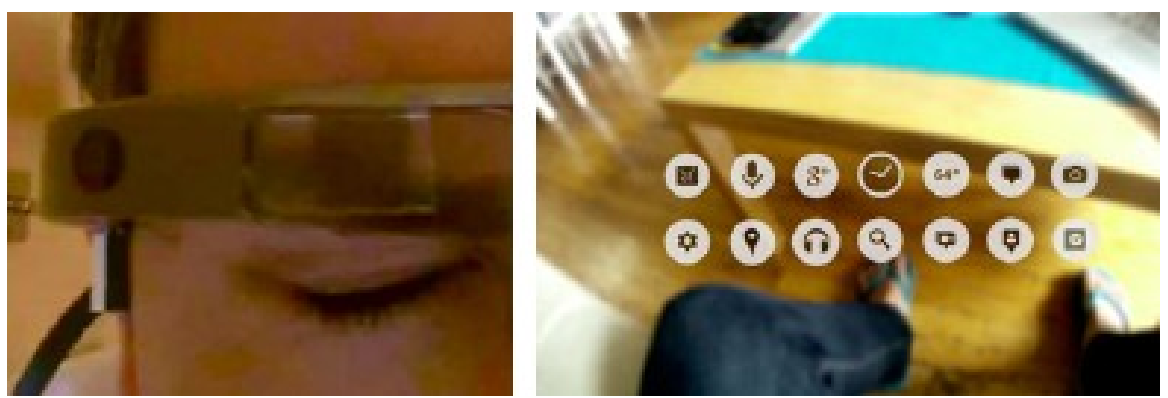

Fig.2: Google glasses technology

Google glasses is equipped with an advanced intelligent mobile phone in the glasses, its different goes through a miniature display screen, which can obtain the augmented reality experience, its core components are in the upper right corner of the frame thick part, there are placed side by side a miniature display screen, one is 720 picture quality of camera, using the glasses can be 3D scanning in real time, and the use of eye can quickly capture the aerobics movement to carry on multiple tag for the aerobics image as shown in Figure 3. 


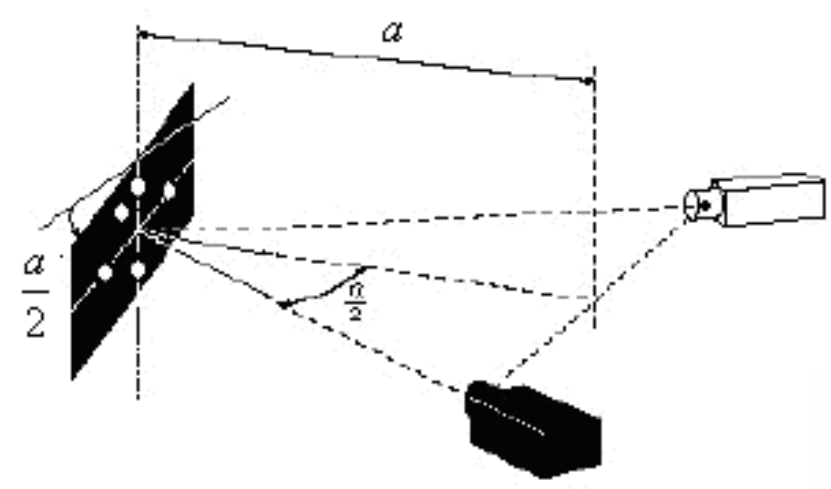

Fig.3: Schematic diagram of multiple calibrations

In order to realize the multi image angle scanning, scanning head can carry out the multiple calibrations to improve the image of the multidimensional performance [12-14]. After scanning head is processed, it need to scan parameter settings, because setting a good parameters can be accurately to the next work as shown in Figure 4, we define a parameter.

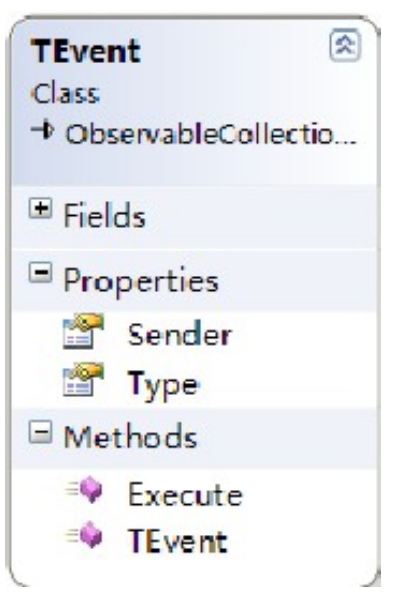

Fig.4: TEvent definition

As shown in Figure 4, it shows the define an image scan event, event support is an important characteristic of visualization, it is different from ordinary.Net control events, because visual control event is actually a inherited read-only attribute from the TEvent class, finally through this property to simulate the.Net event.
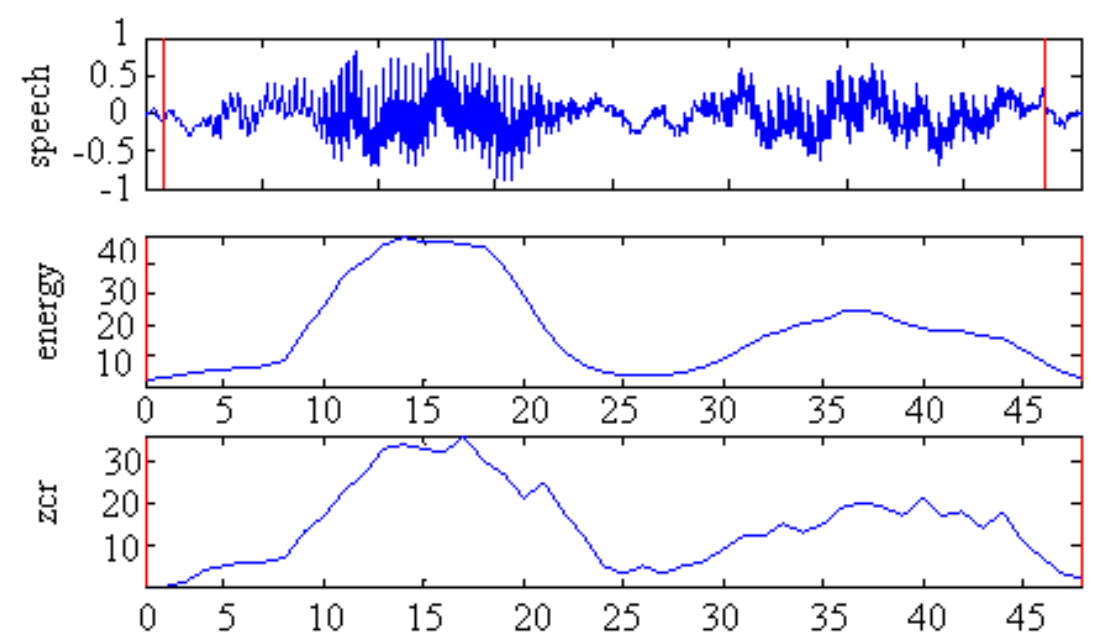

Fig.5: Image data handling

Figure 5 shows the image discrete quantization process, through the amplification of the first image continuous signal, we take a action concentrated element to carry on the main analysis, so as to get the details the aerobics movement and realize the image real time calibration and quantification [15]. The main parameter calculation process is shown in Table 1. 
Table 1: The results of image processing under the condition of no cache

\begin{tabular}{|c|c|c|c|c|}
\hline $\begin{array}{l}\text { Concurrent } \\
\text { number }\end{array}$ & $\begin{array}{l}45 \text { (no } \\
\text { cache) }\end{array}$ & $\begin{array}{l}\text { 90(no } \\
\text { cache) }\end{array}$ & $\begin{array}{l}\text { 150(no } \\
\text { cache) }\end{array}$ & $\begin{array}{l}\text { 240(no } \\
\text { cache) }\end{array}$ \\
\hline Data set & 168.00 & 175.00 & 179.00 & 222 \\
\hline $\begin{array}{l}\text { Picture } \\
\text { stimulation }\end{array}$ & 5 & 5 & 3.5 & 5 \\
\hline Average time & 94.08 & 85.05 & 77.88 & 81.48 \\
\hline Maximum time & 920 & 985 & 1002 & 968 \\
\hline
\end{tabular}

Table 1 shows a series of parameters in no cache conditions produced by image processing, it can be seen from table 1 that picture throughput is very low, it meets the requirement of picture fidelity in the real-time processing process, the average time spent is less, and processing timeliness is good.

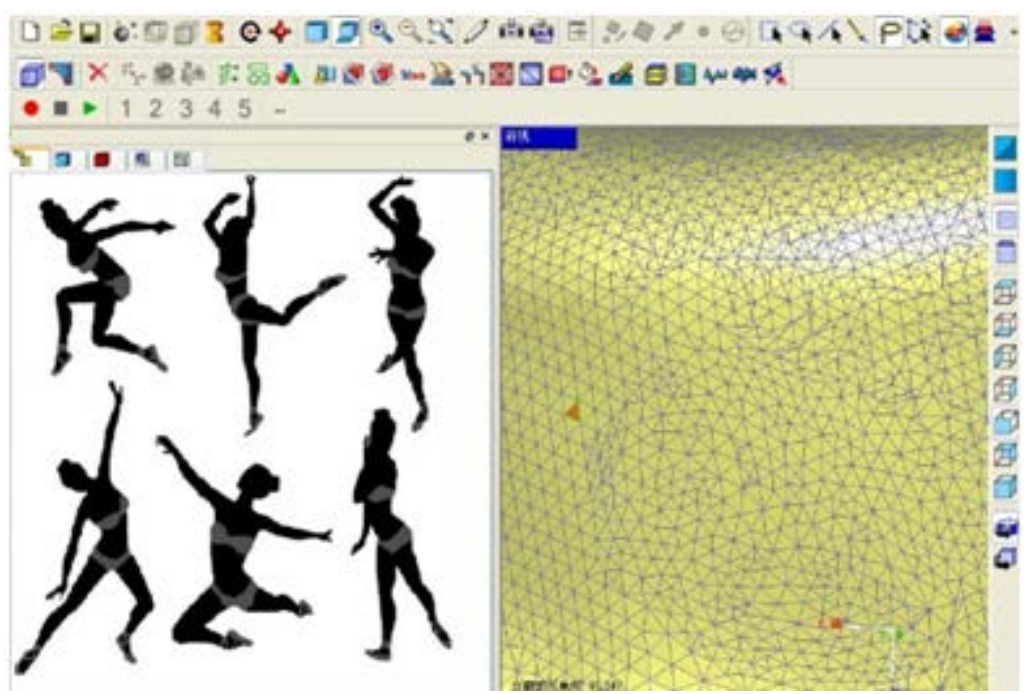

Fig.6: Aerobics image processing

Figure 6 shows the results of aerobics image quantization processing. It can be seen from the chart that after quantization processing, aerobics technical continuity action is retained a few action elements, and the key elements are shown by the form of the triangular cloud, so as to analyze the action angle and art, which provide art materials database for the design of aerobics actions.

\section{Summary}

(1) Design of an new image processing algorithm, the algorithm uses Apriori correlation and multiple calibration quantification as the basis, and then the frequent itemsets of action elements are obtained by relevance search, at the same time using candidate item links can eliminate throughput impact.

(2) Through the aerobics videos and continuity image processing, we found that image throughput is lower, its timeliness and fidelity effect are better, and it can carry out quantify for the image retaining key technical action elements, so as to real-time analyze technical action elements, which is a new computer method in the construction of aerobics action design material library.

\section{References}

[1] G.Q. He. Analysis of competitive aerobics collective six group formation use and changes. Liaoning sports science sports, 2011(5): 96-99.

[2] R.Q. Chen, K.L. Wang. Analysis of aerobics world championships each item difficulty movement in 9 to 10 session. China Sports Science and technology, 2012(5): 112-115.

[3] L. Yang. Prospect of international aerobics 2009 edition rules change characteristics and its competitive sports. Journal of Guangzhou Sports University, 2011(4): 52-55

[4] S.L. Xiao, H. Xu. High level aerobics athletes pay attention to study on removal characteristics of transient. Zhejiang sports science, 2011(4): 75-78 
[5] X.X. Ma. Research on the aesthetic forms of new media installation art - form aesthetics. Popular literature, 2011(23): 56-59.

[6] X.N. Cheng, Y.R. Yang, H.P. Wu. Study of competitive aerobics forgetting process. Journal of Shandong Sports Institute, 2011(1): 35-38.

[7] S. Yan, H.P. Wu. The theory construction of Athlete competition psychological risk. Sports and science, 2012(1): 34-36.

[8] Q.H. Xia. The development trend of aerobics upper limbs action diversity from the tenth world aerobics championship. Zhejiang sports science, 2012(1): 61-65.

[9] F.Y. Chen, Y. Feng, H.B. Li. Influence factor of competitive aerobics players art expressive force. Journal of physical education, 2011(12): 45-46.

[10] C.J. Yu, F.M. Li. Research on our competitive aerobics difficulty and arrangement innovation development under the guidance of the new cycle international aerobics rules in 2009. Journal of Xi'an Physical Education University. 2011(3): 65-68

[11] Y.G. Guan. Reverse engineering data processing technique based on Geomagic Studio. Technology Innovation Herald, 2011(33): 39-44.

[12] Y. Liu, Z.F. Hong, J.B. Qin. Study on 3D modeling technology based on graphics and image. Digital technology and application, 2011(5): 96-99.

[13] L. Gao. The overview of information visualization. Industry and Technology forum, 2011(8): 121-122.

[14] J. Qiao, J.H. Guo, T.L. Lan. The 3D data model of Using the picture modeling technology reconstruction cultural relics. The protection of cultural relics and Archaeological Science, 2012(1): 67-79.

[15] M. Hua, X. Chen, W.C. Wang. The main texture and simple extraction system. Journal of computer aided design and computer graphics, 2011(1): 69-71. 